\title{
Plataforma web de simulación de fenómenos físicos
}

\author{
Fecha de recepción: 12-01-2021 • Fecha de aceptación: 03-02-2021 • Fecha de publicación:10-02-2021
}

Ángel Eduardo Gualán Lozano

Soft Service Plus S.c.c., Ecuador. agualan@datalife.com.ec https://orcid.org/0000-0001-9100-9024

\section{RESUMEN}

El presente trabajo de investigación se centra en desarrollar una plataforma web para simular el fenómeno físico del movimiento parabólico para mejorar las estrategias de enseñanza - aprendizaje en instituciones de educación superior. Se utilizó la metodología SCRUM como marco de desarrollo para la gestión de proyectos y desarrollo ágil de software, mediante esta se pudo obtener el producto final. Tras realizar varias pruebas funcionales se pudo obtener datos estadísticos que permiten deducir que el prototipo web depende del usuario final, en cómo interpreta los datos de los ejercicios planteados y cómo ingresa los mismos como valores de entrada para que la aplicación internamente pueda procesar información y presentar resultados finales trazando la parábola que simula el movimiento deseado.

\section{PALABRAS CLAVE: plataforma web, fenómeno físico, movimiento parabólico, prototipo.}

\section{ABSTRACT}

This research work focuses on developing a web platform to simulate the physical phenomenon of parabolic motion to improve teaching-learning strategies in higher education institutions. The SCRUM 
methodology was used as a development framework for project management and agile software development, by means of which the final product was obtained. After performing several functional tests, it was possible to obtain statistical data that allow us to deduce that the web prototype depends on the end user, on how he/she interprets the data of the proposed exercises and how he/she enters them as input values so that the application can internally process information and present final results by tracing the parabola that simulates the desired movement. 


\section{Introducción}

En la actualidad, las instituciones de educación superior se encuentran en etapas de constante crecimiento, las mismas que generan cambios drásticos en las estrategias del proceso de aprendizaje y enseñanza. Mientras la tecnología avanza, el conocimiento y uso de esta nos lleva a un contexto que está en constante evolución y es por ello que, contar con las herramientas necesarias y saberlas utilizar, hace formar parte de una sociedad globalizada a la vanguardia de la innovación. El desarrollo tecnológico genera la necesidad de transformación de las universidades para que sean capaces de responder a estos cambios y, a la vez, dar respuesta a las necesidades del uso de aplicaciones tecnológicas que permitan fortalecer la educación superior, y colaborar directamente con el desarrollo del país (Velasco \& Carlos, 2017).

La tecnología lleva el proceso de enseñanza a otro nivel, ya que se utiliza distintas aplicaciones que hacen de este proceso más atractivo, tanto para el docente, como para el estudiante, desde un sin número de aplicaciones web, móviles y de escritorio que están disponibles en Internet. Por otro lado, también se puede mencionar que varios académicos han realizado estudios o propuesto el desarrollo de aplicaciones que mejoran el proceso de enseñanza, inicialmente se va a mencionar a Almeida Campos et al. (1997) que realizan una aproximación a la evolución de la enseñanza asistida por computadoras, que se puede decir es la base para el inicio de esta era digital referente a las aplicaciones de enseñanza, por citar ejemplos de este tipo de aplicaciones, se tiene el proceso de enseñanza de matemáticas utilizando el software geogebra (Reisa, 2010), donde se han analizado las contribuciones y los beneficios de esta herramienta en la enseñanza de la asignatura mencionada. Existen aplicaciones de enseñanza que utilizan otras técnicas para llamar la atención del estudiante, Toasa et al. (2019) proponen el desarrollo de una aplicación que utiliza técnicas de gamificación orientada a niños, simulando ser un juego pero que a su vez permite que estos aprendan.

Por lo anterior expuesto, las instituciones de educación superior también requieren de apelaciones que mejoren el proceso de enseñanza, las applets o mini aplicaciones posibilitan realizar simulaciones y de esta manera permiten al estudiante entender mejor las traslaciones entre las diversas representaciones del fenómeno estudiado, las simulaciones permiten explorar elementos de inmersión y de juego para promover el aprendizaje y la participación de los estudiantes en la enseñanza de conceptos sobre colisión de cuerpos (Kercher Greis et al., 2013).

Conociendo los antecedentes mencionados nace la idea de desarrollar una plataforma web que permita realizar simulaciones de procesos y fenómenos físicos, para ser exactos, específicamente este trabajo se enfoca en el movimiento parabólico (Movimiento Parabólico - SiPAQ, n.d.). El applet o mini aplicación se ejecuta dentro de una página web, y vienen representados por una pantalla gráfica que contiene una animación que muestra los resultados analíticos y gráficos con el fin de observar y analizar las consecuencias y cambios que tienen estos fenómenos sobre el proceso en estudio. 


\section{Metodología}

Para desarrollar el proyecto se utilizó el método de investigación deductivo (Abreu, 2014), mediante la cual se pudo realizar un análisis desde lo general hacia lo particular, para luego llegar a una conclusión de la investigación.

\subsection{Análisis y deducción de metodologías de gestión de proyectos y desarrollo de software}

En la Tabla 1 se muestra las diferencias entre los métodos tradicionales y ágiles (Electronics -AGILE - Agile Software Technologies, n.d.) en la gestión de proyectos y desarrollo de software, de esta manera se sustenta la elección del método ágil que se adapta para el desarrollo del trabajo de investigación.

Tabla 1.

Análisis de metodologías agiles vs metodologías tradicionales

\begin{tabular}{|l|l|}
\hline Metodologías Agiles & Metodologías Tradicionales \\
\hline Constante cambios durante el proyecto & Resistencia en los cambios durante el proyecto \\
\hline Menor control en los procesos & Se tiene procesos con políticas y normas \\
\hline No hay contratos & Se tiene un contrato \\
\hline El quipo de desarrollo forma parte del cliente & Se utiliza reuniones para que el cliente interactúe \\
\hline Pocos roles & Mas roles \\
\hline Menos énfasis en la arquitectura del software & $\begin{array}{l}\text { La arquitectura del software es esencial y se } \\
\text { expresa mediante modelos }\end{array}$ \\
\hline $\begin{array}{l}\text { Conclusión: según las premisas generales entre } \\
\text { las metodologías agiles y tradicionales se puede } \\
\text { concluir que una metodología ágil se adapta } \\
\text { como marco de gestión de proyectos y desarrollo } \\
\text { de software para la presente investigación. }\end{array}$ & \\
\hline
\end{tabular}

Fuente: elaboración propia

Existen varias metodologías ágiles de gestión de proyectos y desarrollo de software, en esta investigación se realizará un análisis de las 3 más utilizadas con sus características descritos, tal como se puede ver en la Tabla 2 (Amaya Balaguera, 2015). 
Tabla 2.

Análisis de metodologías agiles más utilizadas

\begin{tabular}{|c|c|c|}
\hline SCRUM & $\mathbf{X P}$ & KANBAN \\
\hline $\begin{array}{l}\text { Trabaja con el ciclo de vida } \\
\text { iterativo e incremental, donde } \\
\text { se va liberando el producto } \\
\text { por pares de forma periódica, } \\
\text { aplicando las buenas prácticas } \\
\text { de trabajo colaborativo }\end{array}$ & $\begin{array}{l}\text { Basada en un conjunto de } \\
\text { reglas y buenas prácticas para } \\
\text { el desarrollo de software en } \\
\text { ambientes muy cambiantes } \\
\text { con requisitos imprecisos, } \\
\text { por ende, está enfocada en la } \\
\text { retroalimentación continua entre } \\
\text { el equipo de desarrollo y el } \\
\text { cliente. }\end{array}$ & $\begin{array}{l}\text { Marco de trabajo que requiere } \\
\text { una comunicación en tiempo } \\
\text { real sobre la capacidad del } \\
\text { equipo, utilizado para controlar } \\
\text { el avance de trabajo en una } \\
\text { línea de producción }\end{array}$ \\
\hline Planifica la cantidad de trabajo & $\begin{array}{l}\text { Desarrollo iterativo e } \\
\text { incremental }\end{array}$ & $\begin{array}{l}\text { Simplifica la asignación de } \\
\text { responsabilidades }\end{array}$ \\
\hline $\begin{array}{l}\text { Retroalimentación mediante } \\
\text { reuniones }\end{array}$ & Programación en parejas & Planificación de tareas \\
\hline Revisión al finalizar cada sprint & Corrección periódica de errores & Métricas visuales \\
\hline Posibles acciones de mejora & $\begin{array}{l}\text { Simplicidad y propiedad del } \\
\text { código compartido }\end{array}$ & Entregas continuas \\
\hline $\begin{array}{l}\text { Conclusión: de acuerdo a } \\
\text { un análisis general de sus } \\
\text { características, de las tres } \\
\text { metodologías agiles más } \\
\text { utilizadas, se pudo deducir que } \\
\text { SCRUM se adapta como marco } \\
\text { de desarrollo para la gestión de } \\
\text { proyectos y desarrollo ágil de } \\
\text { software. }\end{array}$ & & \\
\hline
\end{tabular}

Fuente: elaboración propia

\subsection{Deducción conceptos del movimiento parabólico}

- Premisa 1: composición de movimientos en dos dimensiones en el eje horizontal y vertical.

- Conclusión: para calcular velocidades y distancias recorridas se necesita un plano cartesiano para determinar sus valores tanto el eje $\mathrm{X}$ e $\mathrm{Y}$

- Premisa 2: si el eje $X$ es movimiento rectilíneo uniforme y el eje Y es movimiento rectilíneo uniforme acelerado.

- Conclusión: se necesita conocer los tiempos parciales o totales para completar las fórmulas en las dos dimensiones tanto en el eje $\mathrm{X}$ como en el eje $\mathrm{Y}$.

- Premisa 3: el movimiento parabólico es una composición vectorial, entonces la velocidad forma un ángulo con los componentes del eje $\mathrm{X}$ e $\mathrm{Y}$

- Conclusión: se puede deducir que se necesita conocer de funciones trigonométricas para determinar el ángulo y calcular las velocidades iniciales en los ejes $\mathrm{X}$ e $\mathrm{Y}$. 


\subsection{Aplicación de la metodología ágil deducida}

De acuerdo al método de investigación aplicada con la cual se definió la metodología SCRUM (SCRUM - Proyectos Ágiles, n.d.), como marco de desarrollo para la gestión de proyectos y desarrollo ágil de software, se procede con las fases de desarrollo para cumplir con las funcionalidades del simulador del movimiento parabólico, basados en los conceptos deducidos según las premisas detalladas en el ítem anterior.

Los requisitos que el usuario solicita en el desarrollo están plasmados en las listas de requerimientos funcionales y no funcionales mostrados a continuación.

\section{Funcionales}

RF01: calcular las velocidades iniciales en los ejes $\mathrm{X}$ e $\mathrm{Y}$

RF02: calcular el tiempo de vuelo total del proyectil lanzado.

RF03: calcular la distancia recorrida en los ejes $\mathrm{X}$ e $\mathrm{Y}$

RF04: dibujar una gráfica que indique cual es la trayectoria recorrida del proyectil lanzado.

RF05: el prototipo web debe generar un archivo PDF que permita descargar los resultados calculados

\section{No funcionales}

\section{Producto}

RNF01: no debe tener restricciones de uso para ningún usuario, por tal motivo no es necesario implementar login o control de acceso.

RNF02: presenta resultados finales en base a la deducción previa de los datos que ingrese el usuario final.

Organizacionales

RNF04: ser desarrollado en el lenguaje de programación JavaScript.

RNF05: no debe almacenar información en ninguna base de datos de las simulaciones realizadas.

RNF06: debe permitir realizar simulaciones en diferentes resoluciones de pantallas incluso en navegadores de dispositivos móviles.

En la siguiente Tabla 3 se presentan las características de los usuarios finales del prototipo web. 
Tabla 3.

Perfiles de usuario

\begin{tabular}{|c|c|c|c|}
\hline Nombre de Usuario & Tipo de Usuario & Área Funcional & Actividad \\
\hline Usuario Final. & Administrador & Administración & $\begin{array}{l}\text { Calular los valores finales de: } \\
\text { - } \quad \text { Velocidad Inicial. } \\
\text { - } \quad \text { Velocidad Inicial en X e Y. } \\
\text { - } \quad \text { Tiempo en alcanzar la altura máxima. } \\
\text { - } \quad \text { Tiempo total de vuelo } \\
\text { - } \quad \text { Ángulo de elevación. } \\
\text { - } \quad \text { Distancia total recorrido. } \\
\text { - } \quad \text { Altura máxima alcanzada. } \\
\text { Observar la gráfica final del proyectil } \\
\text { recorrido } \\
\text { Descargar archivo PDF con resumen se la } \\
\text { simulación. }\end{array}$ \\
\hline
\end{tabular}

Fuente: elaboración propia

\section{Resultados}

Cumpliendo con las fases de desarrollo, según SCRUM se requiere documentación necesaria para la ejecución del proyecto.

En cuanto al diseño general, en la Figura 1 se evidencian las personas y roles

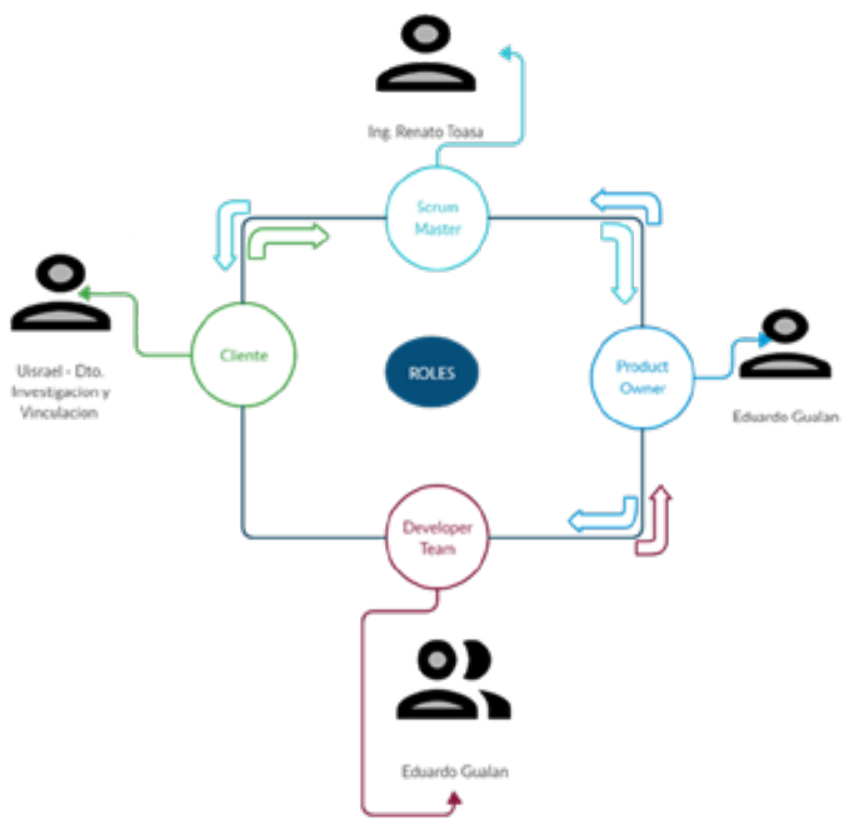

Figura 1. Personas y Roles

Fuente: elaboración propia 
En la Tabla 4 se muestra una lista ordenada de todas las cosas que se deben realizar para cumplir con un proyecto.

Tabla 4.

Product Backlog

\begin{tabular}{|c|c|c|c|c|c|c|c|}
\hline \begin{tabular}{|l|} 
Identificador \\
(ID) de la \\
Historia \\
\end{tabular} & $\begin{array}{l}\text { Enunciado de la } \\
\text { Historia }\end{array}$ & Alias & Estado & Esfuerzo & $\begin{array}{l}\text { Iteración } \\
\text { (Sprint) }\end{array}$ & Prioridad & $\begin{array}{l}\text { Comen- } \\
\text { tarios }\end{array}$ \\
\hline HU01 & $\begin{array}{l}\text { Como usuario } \\
\text { final se necesita } \\
\text { encontrar los } \\
\text { valores de la } \\
\text { velocidad inicial } \\
\text { en } X \text { e Y para } \\
\text { utilizar en las } \\
\text { demás formulas } \\
\text { del movimiento } \\
\text { parabólico. }\end{array}$ & $\begin{array}{l}\text { Velocidad } \\
\text { inicial en } X \\
\text { e Y }\end{array}$ & PLANIFICADO & 3 & 1 & ALTA & \\
\hline $\mathrm{HU} 02$ & $\begin{array}{l}\text { Como usuario } \\
\text { final se desea } \\
\text { determinar el valor } \\
\text { total del tiempo de } \\
\text { vuelo para utilizar } \\
\text { en las demás } \\
\text { formulas del tiro } \\
\text { parabólico } \\
\end{array}$ & $\begin{array}{l}\text { Tiempo total } \\
\text { de vuelo }\end{array}$ & PLANIFICADO & 5 & 1 & ALTA & \\
\hline HU03 & $\begin{array}{l}\text { Como usuario } \\
\text { final se requiere } \\
\text { encontrar la } \\
\text { distancia recorrido } \\
\text { en Y, en cualquier } \\
\text { instante del tiempo } \\
\text { para realizar la } \\
\text { gráfica vertical de } \\
\text { la trayectoria del } \\
\text { proyectil lanzado }\end{array}$ & \begin{tabular}{|l} 
Distancia \\
recorrida \\
en Y
\end{tabular} & PLANIFICADO & 8 & 1 & ALTA & \\
\hline HU04 & $\begin{array}{l}\text { Como usuario } \\
\text { final se necesita } \\
\text { encontrar la } \\
\text { distancia recorrido } \\
\text { en } X \text {, en cualquier } \\
\text { instante del tiempo } \\
\text { para realizar la } \\
\text { gráfica horizontal } \\
\text { de la trayectoria } \\
\text { del proyectil } \\
\text { lanzado } \\
\end{array}$ & \begin{tabular}{|l|} 
Distancia \\
recorrida \\
en X
\end{tabular} & PLANIFICADO & 13 & 1 & ALTA & \\
\hline HU05 & $\begin{array}{l}\text { Como usuario final } \\
\text { se desea graficar } \\
\text { la trayectoria del } \\
\text { proyectil lanzado } \\
\text { para analizar los } \\
\text { valores calculados. }\end{array}$ & $\begin{array}{l}\text { Graficar la } \\
\text { trayectoria } \\
\text { del proyectil }\end{array}$ & PLANIFICADO & 21 & 2 & ALTA & \\
\hline
\end{tabular}

Fuente: elaboración propia 
Mientras que en la Tabla 5 se puede ver una lista de actividades identificadas por el equipo SCRUM, los mismos que se convierten en pequeños entregables.

Tabla 5.

Sprint Backlog

\begin{tabular}{|c|c|c|c|c|c|c|}
\hline $\begin{array}{l}\text { Identificador } \\
\text { (ID) de item } \\
\text { de product } \\
\text { backlog }\end{array}$ & $\begin{array}{l}\text { Enunciado del } \\
\text { item de Product } \\
\text { Backlog }\end{array}$ & Tarea & $\begin{array}{l}\text { Dueño / } \\
\text { Voluntario }\end{array}$ & Estatus & $\begin{array}{l}\text { Horas } \\
\text { estimadas } \\
\text { totales }\end{array}$ & Entregables \\
\hline \multirow[t]{4}{*}{ HU001 } & \multirow{4}{*}{$\begin{array}{l}\text { Como usuario } \\
\text { final se necesita } \\
\text { encontrar los } \\
\text { valores de la } \\
\text { velocidad inicial } \\
\text { en X e Y para } \\
\text { utilizar en las } \\
\text { demás formulas } \\
\text { del movimiento } \\
\text { parabólico. }\end{array}$} & $\begin{array}{l}\text { Determinar la } \\
\text { fórmula para } \\
\text { encontrar la } \\
\text { velocidad inicial } \\
\text { en X }\end{array}$ & $\begin{array}{l}\text { Ángel } \\
\text { Gualán }\end{array}$ & INICIAR & 2 & \multirow[t]{4}{*}{$\begin{array}{l}\text { valor de la } \\
\text { velocidad } \\
\text { inicial en el } \\
\text { eje } \mathrm{X} \text { e } \mathrm{Y}\end{array}$} \\
\hline & & $\begin{array}{l}\text { Definir la fórmula } \\
\text { para encontrar la } \\
\text { velocidad inicial } \\
\text { en } Y\end{array}$ & $\begin{array}{l}\text { Ángel } \\
\text { Gualán }\end{array}$ & INICIAR & 2 & \\
\hline & & $\begin{array}{l}\text { Programar la } \\
\text { función halla } \\
\text { velocidad inicial } \\
\text { en } X\end{array}$ & $\begin{array}{l}\text { Ángel } \\
\text { Gualán }\end{array}$ & INICIAR & 4 & \\
\hline & & $\begin{array}{l}\text { Desarrollar la } \\
\text { función halla } \\
\text { velocidad inicial } \\
\text { en Y }\end{array}$ & $\begin{array}{l}\text { Ángel } \\
\text { Gualán }\end{array}$ & INICIAR & 4 & \\
\hline \multirow[t]{2}{*}{ HU002 } & \multirow{2}{*}{$\begin{array}{l}\text { Como usuario } \\
\text { final se desea } \\
\text { determinar el } \\
\text { valor total del } \\
\text { tiempo de vuelo } \\
\text { para utilizar } \\
\text { en las demás } \\
\text { formulas del tiro } \\
\text { parabólico }\end{array}$} & $\begin{array}{l}\text { Determinar la } \\
\text { fórmula para } \\
\text { encontrar el tiempo } \\
\text { de vuelo }\end{array}$ & $\begin{array}{l}\text { Ángel } \\
\text { Gualán }\end{array}$ & INICIAR & 2 & \multirow[t]{2}{*}{$\begin{array}{l}\text { Valor del } \\
\text { tiempo de } \\
\text { vuelo del } \\
\text { proyectil }\end{array}$} \\
\hline & & $\begin{array}{l}\text { Programar la } \\
\text { función calcular } \\
\text { tiempo total de } \\
\text { vuelo }\end{array}$ & $\begin{array}{l}\text { Ángel } \\
\text { Gualán }\end{array}$ & INICIAR & 4 & \\
\hline \multirow[t]{2}{*}{ HU003 } & \multirow{2}{*}{$\begin{array}{l}\text { Como usuario } \\
\text { final se requiere } \\
\text { encontrar } \\
\text { la distancia } \\
\text { recorrido en Y, en } \\
\text { cualquier instante } \\
\text { del tiempo para } \\
\text { realizar la gráfica } \\
\text { vertical de la } \\
\text { trayectoria del } \\
\text { proyectil lanzado }\end{array}$} & $\begin{array}{l}\text { Definir la fórmula } \\
\text { para encontrar la } \\
\text { distancia recorrida } \\
\text { en } Y\end{array}$ & $\begin{array}{l}\text { Ángel } \\
\text { Gualán }\end{array}$ & INICIAR & 2 & \multirow[t]{2}{*}{$\begin{array}{l}\text { Valor de la } \\
\text { distancia } \\
\text { recorrido en } \\
\text { el eje Y }\end{array}$} \\
\hline & & $\begin{array}{l}\text { Desarrollar la } \\
\text { función distancia } \\
\text { recorrido en } Y\end{array}$ & $\begin{array}{l}\text { Ángel } \\
\text { Gualán }\end{array}$ & INICIAR & 4 & \\
\hline
\end{tabular}




\begin{tabular}{|c|c|c|c|c|c|c|}
\hline \multirow[t]{2}{*}{ HU004 } & \multirow{2}{*}{$\begin{array}{l}\text { Como usuario } \\
\text { final se necesita } \\
\text { encontrar } \\
\text { la distancia } \\
\text { recorrido en X, en } \\
\text { cualquier instante } \\
\text { del tiempo para } \\
\text { realizar la gráfica } \\
\text { horizontal de la } \\
\text { trayectoria del } \\
\text { proyectil lanzado }\end{array}$} & \begin{tabular}{|l|} 
Determinar la \\
fórmula para \\
encontrar la \\
distancia recorrida \\
en X
\end{tabular} & $\begin{array}{l}\text { Ángel } \\
\text { Gualán }\end{array}$ & INICIAR & 2 & \multirow[t]{2}{*}{$\begin{array}{l}\text { Valor de la } \\
\text { distancia } \\
\text { recorrido en } \\
\text { el eje X }\end{array}$} \\
\hline & & $\begin{array}{l}\text { Programar la } \\
\text { función distancia } \\
\text { recorrido en el } \\
\text { eje X }\end{array}$ & $\begin{array}{l}\text { Ángel } \\
\text { Gualán }\end{array}$ & INICIAR & 4 & \\
\hline \multirow[t]{5}{*}{ HU005 } & \multirow{5}{*}{$\begin{array}{l}\text { Como usuario } \\
\text { final se desea } \\
\text { graficar la } \\
\text { trayectoria del } \\
\text { proyectil lanzado } \\
\text { para analizar } \\
\text { los valores } \\
\text { calculados. }\end{array}$} & $\begin{array}{l}\text { Buscar librería } \\
\text { JavaScript para } \\
\text { realizar gráficos }\end{array}$ & $\begin{array}{l}\text { Ángel } \\
\text { Gualán }\end{array}$ & INICIAR & 4 & \multirow{5}{*}{\begin{tabular}{|l} 
Grafica \\
final de \\
Resultados \\
en la IU y \\
formato PDF
\end{tabular}} \\
\hline & & \begin{tabular}{|l|} 
Diseño del \\
formulario ingreso \\
de datos \\
\end{tabular} & \begin{tabular}{|l|} 
Ángel \\
Gualán
\end{tabular} & INICIAR & 4 & \\
\hline & & \begin{tabular}{|l|} 
Pintar el plano \\
cartesiano en la IU
\end{tabular} & $\begin{array}{l}\text { Ángel } \\
\text { Gualán }\end{array}$ & INICIAR & 6 & \\
\hline & & \begin{tabular}{|l} 
Dibujar la \\
trayectoria del \\
proyectil
\end{tabular} & $\begin{array}{l}\text { Ángel } \\
\text { Gualán }\end{array}$ & INICIAR & 6 & \\
\hline & & $\begin{array}{l}\text { Programar la } \\
\text { función descargar } \\
\text { PDF }\end{array}$ & $\begin{array}{l}\text { Ángel } \\
\text { Gualán }\end{array}$ & INICIAR & 2 & \\
\hline
\end{tabular}

Fuente: elaboración propia

Por otra parte, en la Figura 2 se observa cómo se realizó el desarrollo de cada sprint.

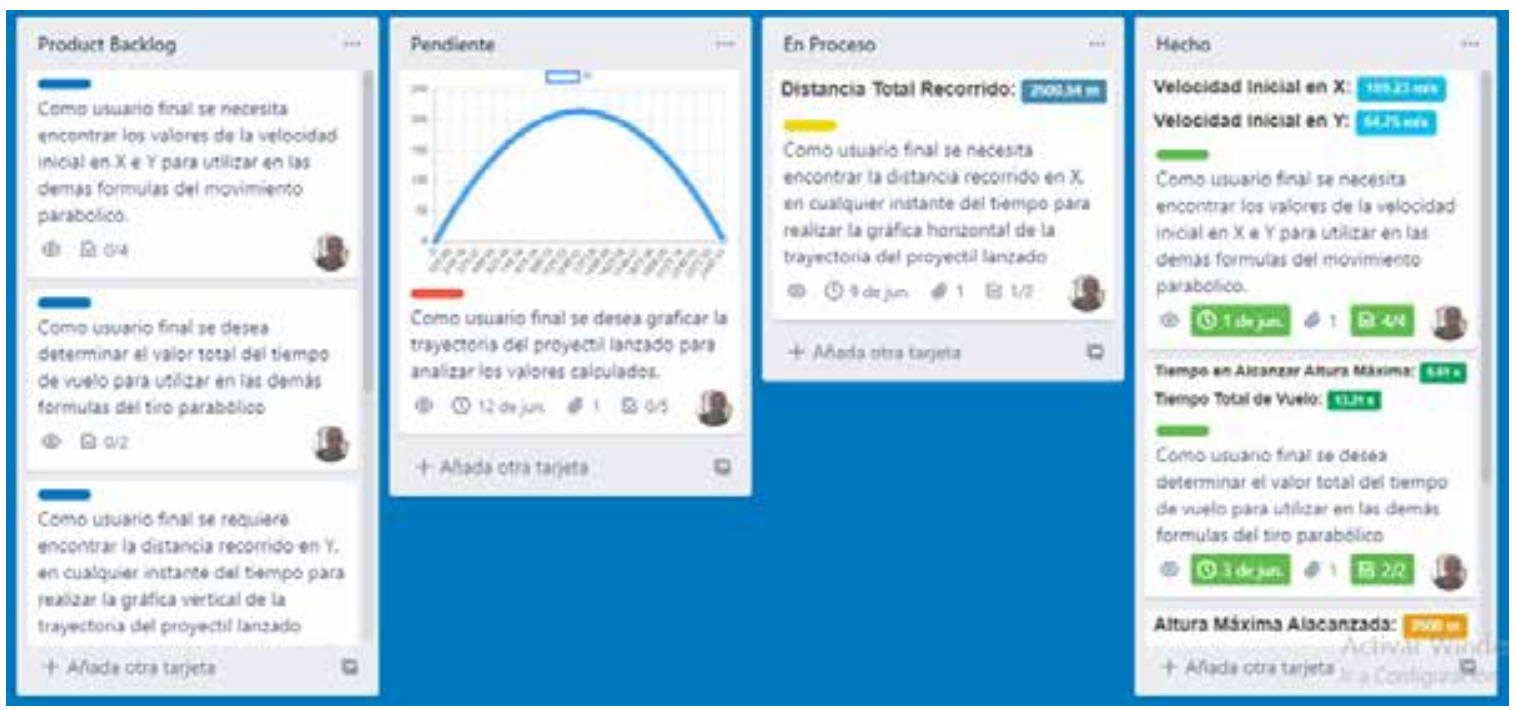

Figura 2. Sprint Desarrollado

Fuente: elaboración propia 
A su vez, el prototipo web se desarrolló con la arquitectura MVC (Díaz González \& Fernández Romero, 2012) como se detalla en el siguiente diagrama representado en la Figura 3.

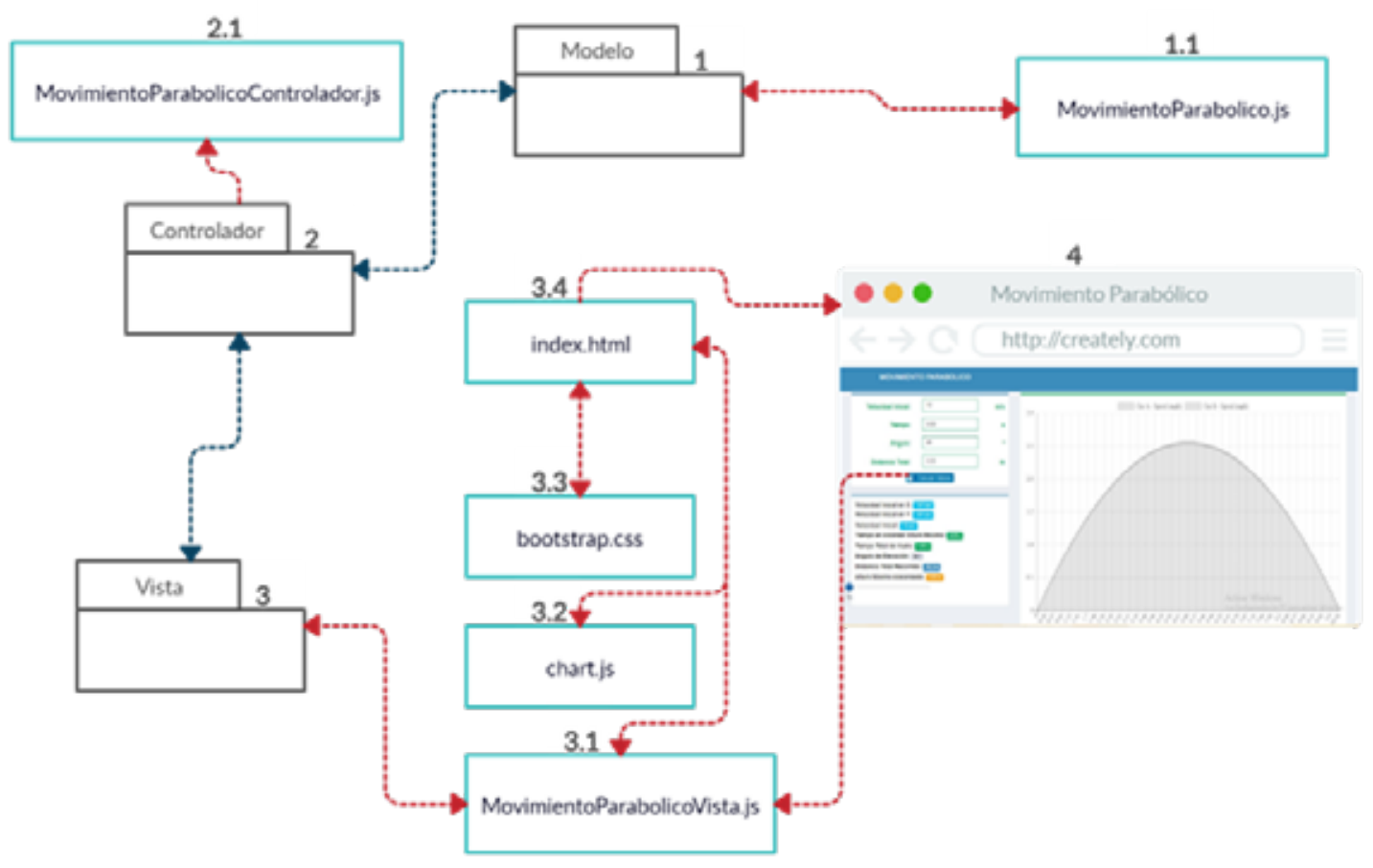

Figura 3. Arquitectura del prototipo web

Fuente: elaboración propia

La arquitectura MVC consiste en separar el prototipo web en tres componentes, el modelo se encarga de gestionar, manipular y actualizar datos, la vista se encarga de mostrar la pantalla al usuario final y el controlador es la parte principal donde se especifican los métodos y funciones del prototipo web.

Como resultado final del presente trabajo de investigación se obtuvo la plataforma web para realizar simulaciones del fenómeno físico movimiento parabólico (Figura 4). El prototipo web actualmente se encuentra publicado en el siguiente subdominio http://movimientoparabolico.netfly.net.ec/ 


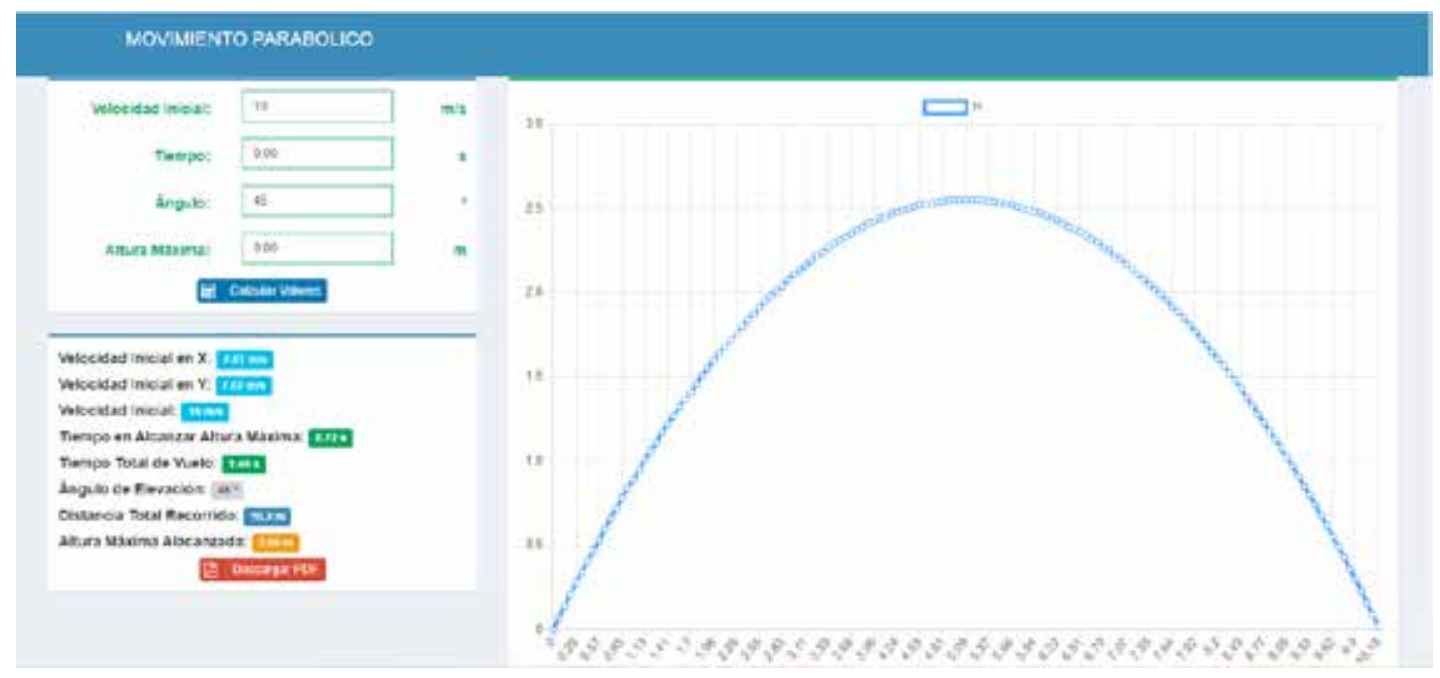

Figura 4. Prototipo web para simular el movimiento parabólico

Fuente: elaboración propia

\subsection{Pruebas realizadas}

Para validar los requerimientos funcionales y no funcionales del prototipo web se realizaron las siguientes pruebas:

a. Pruebas de funcionalidad unitarias: se realizaron las pruebas de todas las funciones programadas que realizan cálculos matemáticos y funciones trigonométricas con diferentes valores e incluso con datos de entrada que no son valores numéricos obteniendo los siguientes resultados finales.

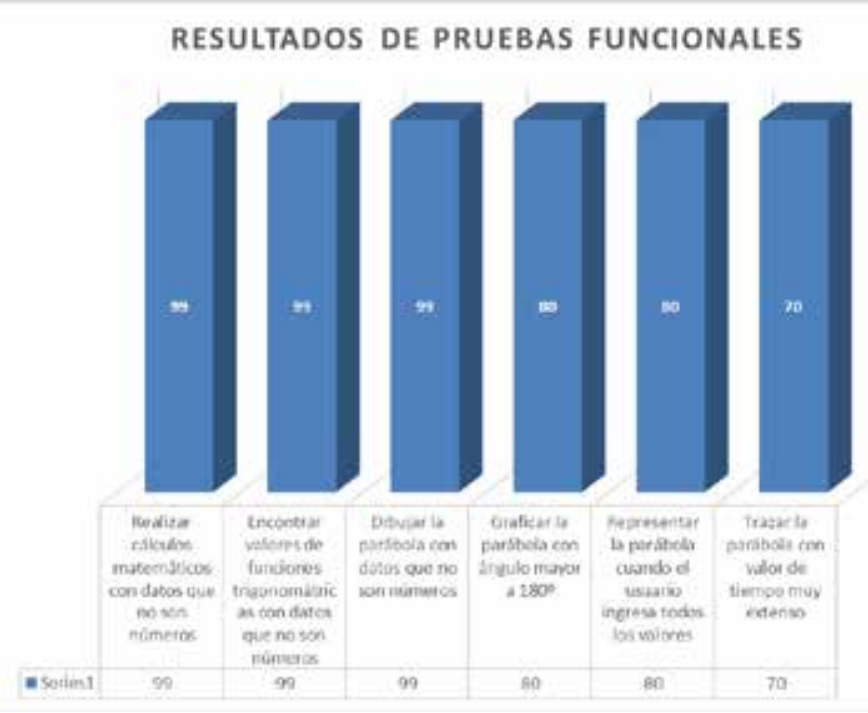

Figura 5. Resultados de pruebas funcionales

Fuente: elaboración propia 
b. Pruebas no funcionales de rendimiento: para realizar dichas pruebas de manera satisfactoria se utilizó la herramienta GTmetrix (Emilio et al., 2015) la cual sirve para realizar test de velocidad y determinar el rendimiento de sitios webs, dicha herramienta fue desarrollada por GT.net una empresa canadiense y el mismo que se encuentra disponible para su uso de manera online, se adjunta el reporte final del prototipo web analizado.

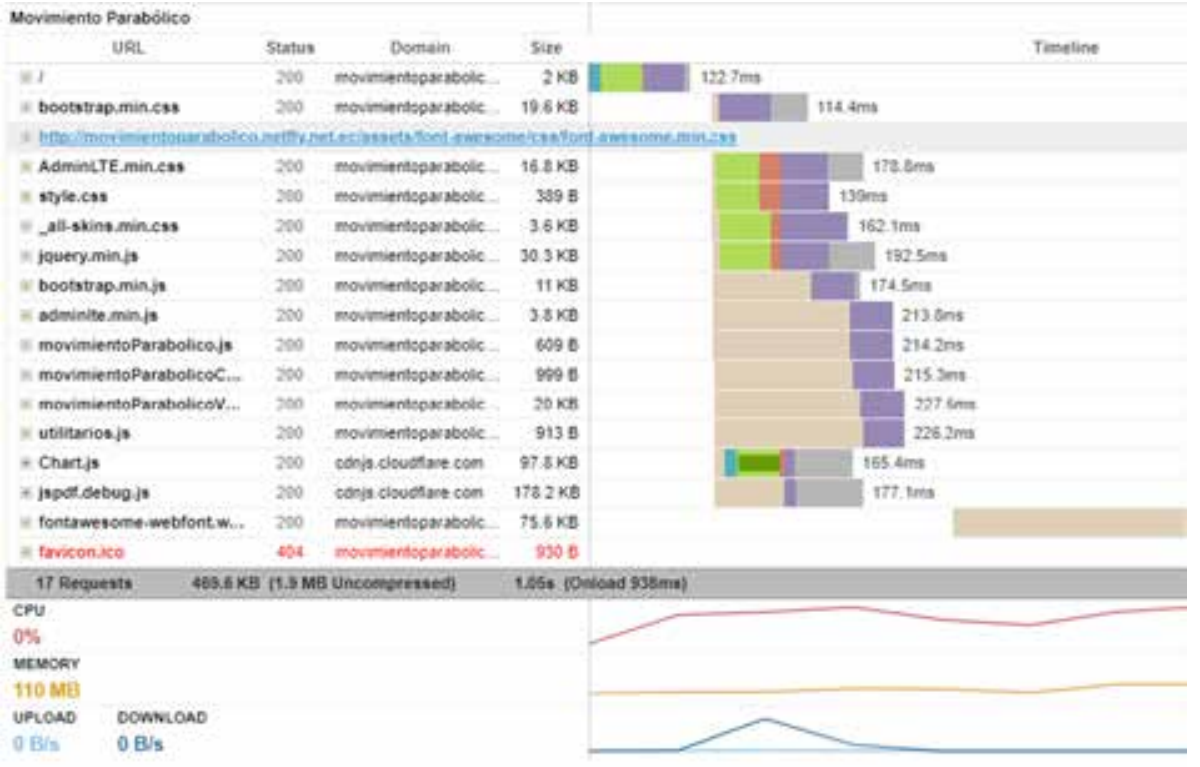

Figura 6. Resultados de pruebas de rendimiento

Fuente: elaboración propia

Según los datos estadísticos de las pruebas realizadas se puede deducir que el prototipo web para su correcto funcionamiento, y sobre todo para trazar la parábola, depende de cómo el usuario final interpreta los datos de los ejercicios planteados y cómo ingresa los mismos como valores de entrada para que la aplicación internamente pueda procesar información y presentar resultados finales. En cuanto a las pruebas de rendimiento no se tiene mayores inconvenientes debido a que el mismo está desarrollado en un lenguaje de programación interpretado y se ejecuta del lado del cliente.

\section{Conclusiones}

Se puede concluir que, en la recopilación de información para levantar requerimientos funcionales y no funcionales del prototipo web, fue indispensable el plan denominado implementación de laboratorios virtuales de física, ya que en dicho archivo PDF se encuentra toda la información necesaria, fases de desarrollo y personas involucradas en el proyecto con la cual se pudo definir el panorama de a dónde se quiere llegar.

Al utilizar tecnologías actuales como HTML5, CSS3 y el Framework de maquetado Bootstrap se facilita el diseño responsive y es adaptable a diferentes resoluciones de pantallas e incluso 
en dispositivos móviles. Además, al utilizar JavaScript como lenguaje de programación se pudo obtener un prototipo web bastante liviano ya que el mismo ejecuta instrucciones directas y libres sin necesidad de compilar. Por lo tanto, se puede concluir que la utilización de estas herramientas facilita el desarrollo web.

Al realizar las pruebas funcionales y de rendimiento del prototipo web, se pudo definir que el mismo depende del usuario final en cómo interpreta los ejercicios planteados y qué datos ingresa en la aplicación para que el mismo pueda cumplir con los procesos necesarios y requeridos para realizar los cálculos matemáticos y presentar valores como resultados finales simulando el movimiento parabólico. 


\section{Referencias}

Abreu, J. L. (2014). El Método de la Investigación Research Method. Daena: International Journal of Good Conscience, 9(3), 195-204.

Almeida Campos, S., Febles Rodríguez, J. P., \& Bolaños Ruiz, O. (1997). Evolución de la enseñanza asistida por computadoras. In Educación Médica Superior (1st ed., Vol. 11, pp. 31-38). http://scielo.sld.cu/scielo. php?script=sci_arttext\&pid=S0864-21411997000100005

Amaya Balaguera, Y. D. (2015). Metodologías ágiles en el desarrollo de aplicaciones para dispositivos móviles. Estado actual. Revista de Tecnología, 12(2). https://doi.org/10.18270/rt.v12i2.1291

Díaz González, Y., \& Fernández Romero, Y. (2012). Patrón Modelo-Vista-Controlador. Telematica, 11(2). https:// www.revistatelematica.cujae.edu.cu/index.php/tele/article/view/15

Electronics -AGILE - Agile Software Technologies. (n.d.). Retrieved January 7, 2021, from http://virtual.vtt.fi/ virtual/agile/mobiled.html

Emilio, G., Flores, V., Dolores, J., Ayala, R., Barraza, E. U., Agundis, D. U., Héctor, L., \& Muñoz, G. (2015). Pruebas Operativas de Carga de Páginas Web Usando GTmetrix. Pistas Educativas, 36(114). http://gtmetrix. com

Kercher Greis, L., Reategui, E., \& Iwaszko Marques, T. B. (2013). Vista de Un Simulador de Fenómenos Físicos para los Mundos Virtuales / A physical phenomena simulator for virtual worlds. Revista Latinoamericana de Tecnología Educativa, 12, 51-62. https://relatec.unex.es/article/view/893/743

Movimiento parabólico - SiPAQ. (n.d.). Retrieved January 20, 2021, from http://sipaq.webs.upv.es/movimiento-parabolico/

Reisa, Z. A. (2010). Computer supported mathematics with Geogebra. Procedia - Social and Behavioral Sciences, 9, 1449-1455. https://doi.org/10.1016/j.sbspro.2010.12.348

SCRUM - Proyectos Ágiles. (n.d.). Retrieved November 26, 2020, from https://proyectosagiles.org/que-esscrum/

Toasa, R., Burbano, E., Constante, A., Hidalgo, L., \& Morales, F. (2019). A Custom and Dynamic Game using Gamification Techniques to Children from 4 to 5 years old. 2019 14th Iberian Conference on Information Systems and Technologies (CISTI), 1-5. https://doi.org/10.23919/CISTI.2019.8760593

Velasco, C., \& Carlos, J. (2017). La Educación Superior en el Ecuador. Situación actual y perspectivas de futuro desde el contexto de las Tecnologías de la Información y la Comunicación. [s.I.]: [s.n.]. http://www.dspace. uce.edu.ec/handle/25000/13671 